\title{
A combination of check tug and fascial interposition with no- scalpel vasectomy
}

\author{
Vivek Kumar, MS, FRCS, Senior SHO-Urology, Leighton Hospital, Crewe, UK \\ Ramchandra M Kaza, MS, Prof. Surgery, Maulana Azad Medical College, Delhi, India
}

Correspondence: Mr Vivek Kumar, MH-79, Leighton Hospital, Crewe, Cheshire, CW1 4QJ, UK. Tel: 01270255141 extn. 2691 bleep: 2422, Fax: 01270 587696, Email: vkumar3908@hotmail.com

(Accepted August $24^{\text {th }}, 2000$ )

\begin{abstract}
Summary
The prospective study was carried out to evaluate the success rates of combining a check tug and fascial interposition while performing a no-scalpel vasectomy.

A total of 2150 male acceptors visiting the family welfare clinic of Lok Nayak Hospital, Delhi, from July 1992 to June 1995 and opting for a no-scalpel vasectomy, underwent a combination of check tug and fascial interposition during the procedure. These men were followed-up for failure rates as shown by semen analysis, 3 months and at least 20 ejaculations following the procedure.

All the clients showed a negative sperm count during the subsequent follow-up, less than two non-motile sperms per high power field being taken as a negative count $(n=5)$ and there were no reported pregnancies.

A check tug ensures that the same vas deferens is not ligated twice, particularly as the approach is a midline one. The fascial interposition prevents spontaneous recanalisation of the cut vas. Thus the combination of the two would further increase the reliability of the procedure.
\end{abstract}

\section{Keywords}

check tug, fascial interposition, midline

\section{Key message points}

- A combination of check tug and fascial interposition with no-scalpel vasectomy reduced early failure rates.

- This combination may increase the long-term reliability of the procedure.

\section{Introduction}

We started no-scalpel vasectomy at Maulana Azad Medical College and associated Lok Nayak Hospital, Delhi in 1989. Once the technique was well established, a prospective study was carried out to know the effectiveness, in terms of failure rate, of this technique when a check tug was combined with fascial interposition.

\section{Method}

A total of 2150 male acceptors of vasectomy visiting the Family Welfare Clinic of Lok Nayak Hospital, Delhi, underwent a combination of check tug and fascial interposition with no-scalpel vasectomy. The period of study was from July 1992 to June 1995.

The operation was performed as standard Li's vasectomy using a midline approach under local anesthesia using $1 \%$ lignocaine infiltration. ${ }^{1}$ A $1 \mathrm{~cm}$ piece of vas deferens was excised. A gentle tug was given to the uncut ligature used to ligate the testicular end of the vas deferens to make sure that the ipsilateral testis was lifted. This maneuver was repeated on the other side, the approach being through the same incision. This ensured that both the vases were ligated.
All the patients were followed-up as per the guidelines for sterilisation issued by the Ministry of Health and Family Welfare, Government of India. ${ }^{2}$ They had their semen analysed 3 months and at least 20 ejaculations following vasectomy. The examination included a preliminary microscopy followed by centrifugation of the sample. An open register was maintained for reported pregnancies and complications.

\section{Results}

There were no cases of failure as demonstrated by a negative sperm count on 3 months post-vasectomy semen analysis, and no reported pregnancy. Less than two non-motile sperms, as were seen in five men, was taken as a negative count.

\section{Discussion}

There was a nil failure rate as defined by our semen testing criteria at 3 months when check tug was combined with fascial interposition. The early failure rates of vasectomy range from $0.5 \%$ to $5 \%$, depending on the method used to occlude the lumen. ${ }^{3}$ The failure can be due to non-identification of either vases, recanalisation, or too few ejaculations. ${ }^{4}$

Since the approach is a midline one, there are chances that the same vas may be ligated twice. The one on the opposite side thus remains patent, resulting in failures. A check tug ensures that this mistake is avoided and it increases the reliability of the procedure by reducing the early failure rates. Philp and associates ${ }^{5}$ have reported delayed recanalisation following vasectomy, the chances being one in 2800. For assessing this late failure, a further prospective study including a larger number of men with longer follow-up is required. A larger follow-up with sequential semen analysis will also help in assessing the problem of transient reappearance of sperms following vasectomy with this technique, that has been observed with conventional vasectomy in a study done at Elliot-Smith Clinic, Oxford. ${ }^{6}$ Though pregnancies have been reported in the past inspite of a negative sperm count, ${ }^{7}$ there have been none in this series.

\section{Conclusion}

The combination of check tug and fascial interposition with no-scalpel vasectomy reduced the early failure rates and may increase the long-term reliability of the procedure.

\footnotetext{
Statements on funding and competing interests

Funding. None.

Competing interests. None.

References

Li S, Goldstein M, Zhu U, et al. The no-scalpel vasectomy, J Urol 1991; 145: 341-344. tandards of male and female sterilization. Department of Family Welfare, Ministry of Health

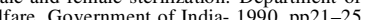

Goldstein M. No scalpel vasectomy. Camphell's Urology, $7^{\text {th }}$ edition. Philadelphia, WB Saunders, 1998: 1340-342.

Rajfer J, Bannett CJ. Vasectomy. Urol Clin North Am 1988; 15 (4): 631-634.

Philp T, Guillebaud J, Budd D. Complication of vasectomy: review of 16000 patients. B J Urol 1984; 56 (6): 745-748.

O'Brien TS, Cranston D, Ashwin P, et al. Temporary reappearance of sperms 12 months after vasectomy clearance. Br J Urol 1995; 76 (3): 371-372.

Smith JC, Cranston D, O'Brien T. Fatherhood without apparent spermatozoa after vasectomy. Lancet 1994; 344 (8919): 415.
} 